\title{
Hydrodynamic responses of a thin floating disk to regular waves
}

\author{
Yiew, L. J. ${ }^{a}$, Bennetts, L. G. ${ }^{a}$, Meylan, M. H. ${ }^{b}$, French, B. J. ${ }^{c}$, Thomas, \\ G. A. ${ }^{\mathrm{c}, \mathrm{d}}$ \\ ${ }^{a}$ School of Mathematical Sciences, University of Adelaide, SA 5005, Australia \\ ${ }^{b}$ School of Mathematical and Physical Science, University of Newcastle, NSW 2308, \\ Australia \\ ${ }^{c}$ National Centre for Maritime Engineering and Hydrodynamics, Australian Maritime \\ College, TAS 7250, Australia \\ ${ }^{d}$ Department of Mechanical Engineering, University College London, WC1E 7JE, U.K.
}

\begin{abstract}
The surge, heave and pitch motions of two solitary, thin, floating disks, extracted from laboratory wave basin experiments are presented. The motions are forced by regular incident waves, for a range of wave amplitudes and frequencies. One disk has a barrier attached to its edge to stop the incident waves from washing across its upper surface. It is shown that the motions of the disk without the barrier are smaller than those of the disk with the barrier. Moreover, it is shown that the amplitudes of the motions, relative to the incident amplitude, decrease with increasing incident wave amplitude for the disk without a barrier and for short incident wavelengths. Two theoretical models of the disk motions are considered. One is based on slope-sliding theory and the other on combined linear potential-flow and thin-plate theories. The models are shown to have almost the same form in the long-wavelength regime. The potential-flow/thinplate model is shown to capture the experimentally measured disk motions with reasonable accuracy.
\end{abstract}

Keywords: Ocean waves, sea ice, marine geophysics

Email address: lucas.yiew@adelaide.edu.au (Yiew, L. J.)

Preprint submitted to Ocean Modelling

September 16, 2015 


\section{Introduction}

As ocean surface waves progress deeper into the partially sea ice covered ocean, they encounter discrete, relatively thin chunks of ice (floes) of increasing horizontal dimensions (Squire and Moore, 1980). The range of dimensions de-

5 pends on the geographic location and season. However, the floe diameters can be as small as a metre, in the case of pancake ice, and up to hundreds of metres. The waves are attenuated by their interactions with the floes before they reach the quasi-continuous ice pack (Shen and Ackley, 1991), notwithstanding the large floes pushed into the outer fringes of the ice cover by random ice motions, which are subsequently broken up by the waves (Squire et al., 1995). The region of the ice-covered ocean in which wave activity remains significant is known as the marginal ice zone (MIZ).

Waves impact the ice cover in the MIz. They break up the ice into smaller floes (Prinsenberg and Peterson, 2011), which are more prone to melting and easily stirred up by winds, currents and waves. For example, waves herd the floes into groups (Wadhams, 1983). Further, waves cause floes to collide with one another (Martin and Becker, 1987), which cause them to erode and produce rubble (McKenna and Croker, 1990). Collisions sometimes turn into rafting events, and if the floes stay in contact they bond (Dai et al. 2004). Waves also introduce warm water and overwash the floes, which accelerates melt (Wadhams et al. 1979, Massom and Stammerjohn, 2010).

Arctic sea ice is retreating polewards, particularly following the summer melt season (Stroeve et al., 2014). Strong and Rigor (2013) use satellite data from 1979 to 2011 to show that, in addition to the poleward shift of the Arctic ice cover, the width of the MIZ is increasing by approximately $13 \mathrm{~km}$ per decade during the summer months. (They defined the MIZ based on the ice covering 15 to $80 \%$ of the ocean surface.) Thomson and Rogers (2014), amongst others, use models and data to show that winds generate large-amplitude waves in the areas of open water left by the ice retreat. Squire (2011), for example, argues that 
weakening the ice cover, accelerating ice retreat and promoting even stronger waves.

Theoretical/numerical models have been developed to predict wave impacts on the ice cover. Shen and Ackley (1991) used a one-dimensional model to 35 study collisions between floes and herding. They used the slope-sliding model of Rumer et al. (1979) to calculate the horizontal motions of the floes induced by waves. The slope-sliding model is an extension of Morison's equation, which includes a force due to the slope of the wave field. The model is derived on the assumption that floes do not modify the wave field, i.e. the floe diameter is much less than the wavelength. It predicts the horizontal motion of a floe to be the sum of an oscillatory surge motion at the period of the incident wave, and a steady drift in the direction of the incident wave. Shen and Zhong (2001) derived analytical solutions to the slope-sliding model in certain cases. Marchenko (1999) independently derived a similar slope-sliding theory

45 to Rumer et al. (1979). Grotmaack and Meylan (2006) related the two theories and identified an error in the derivation of Rumer et al. (1979), although they noted Marchenko (1999) neglected the floe's added mass.

Kohout and Meylan (2008) and Williams et al. (2013a|b) modelled waveinduced breakup of a large group of ice floes, and applied breakup criteria that 50 extended the earlier work of Langhorne et al. (2001). The kernel of both models is a model of a wave interacting with a solitary floe. The wave-floe interaction model uses linear potential-flow theory to model water motions and thin-plate theory to model the floe. The linear potential-flow/thin-plate model is commonly used to study wave-floe interactions (see the review of Squire, 2007, for 55 example).

Kohout and Meylan (2008) and Williams et al. (2013a b) used two-dimensional models (one horizontal dimension and one depth dimension). Masson and LeBlond (1989), Meylan et al. (1997) and Bennetts et al. (2010) developed three-dimensional models of waves propagating through large groups of floes.

${ }_{60}$ They focussed on the attenuation of wave energy into the ice-covered ocean and did not model breakup or any other impact of the waves on the ice cover. 
Masson and LeBlond (1989) and Meylan et al. (1997) modelled the floes using the thin-disk models of Isaacson (1982) and Meylan and Squire (1996), respectively, noting the former is a rigid model and the latter is an elastic model. 65 Bennetts et al. (2010) used a disk model and also a square-plate model, using the finite-element approach of Meylan (2002), but found the different shapes did not significantly alter the predicted attenuation rates.

A handful of laboratory experimental studies have been conducted recently to assess the accuracy of the theoretical models, and indicate phenomena the models do not capture. The experiments focus on the ability of the models to predict interactions between water waves and thin floating plates.

Bennetts and Williams (2015) used laboratory wave basin experiments to validate the model of Meylan et al. (1997), and the two-dimensional model of Bennetts and Squire (2012), which was used by Williams et al. (2013ab).

75 They used arrays of 40 to 80 identical wooden disks to model the ice cover, and measured the proportion of wave energy it transmitted for regular incident waves over a range of wave frequencies and, in two cases, for two different amplitudes. The quotient of thickness, $H$, over diameter, $D$, for the disks was $H / D \approx 3.3 \times 10^{-2}$. The quotient of the incident wavelength, $\lambda$, over the disk diameters was in the range $\lambda / D \approx 0.67$ to 6.28 . The incident steepness, represented by the product $k a$, where $k=2 \pi / \lambda$ is the wavenumber and $a$ is the incident amplitude, was in the range $k a \approx 0.04$ to 0.26 . They showed the models predict the transmitted energy accurately for small incident amplitudes and low concentrations of the disks. They observed the models were inaccurate for the larger incident amplitudes when wave overwash of the disks was strong - overwash is a form of green water that refers to the wave running semi-continuously over the top of the disks, due to their small freeboards. Further, they provided evidence to show the models were inaccurate for high concentrations due to collisions between the disks, caused by out of phase surge motion of adjacent disks, and rafting, caused by out of phase heave and pitch motions. The potential-flow/thin-plate model does not include the highly nonlinear processes of overwash and collisions. 
Modelling collisions between disks requires an accurate model of the surge motion of a solitary disk. Heave and pitch motions must also be modelled to predict rafting. However, the potential-flow/thin-plate and slope-sliding models' predictions of these oscillatory motions have not yet been thoroughly validated.

Bennetts and Williams (2015) presented measured surge, heave and pitch motions of a solitary wooden disk for a subset of the incident frequencies and amplitudes used for their multiple-disk tests, as an addendum to their investigation of wave transmission through multiple disks. They compared the measurements to the predictions of the potential-flow/thin-plate model, and found the model is, in general, accurate. They showed the model was least accurate for a test in which strong overwash occurred. In particular, the model overpredicted the translational motions, surge and heave, and underpredicted the rotational motion, pitch.

Previously, Montiel et al. (2013a b) presented measurements of the flexural motions of a thin plastic disk in response to regular incident waves, as functions of the incident frequency. They used three thin disks, with $H / D=2.1 \times 10^{-3}$ to $6.9 \times 10^{-3}$, and incident waves with lengths ranging from $\lambda / D \approx 0.63$ to 3.14 , and two small steepnesses $k a \approx 0.03$ and 0.06 . They compared the measurements to predictions of the potential-flow/thin-plate model. However, they used a vertical rod through the centre of the disk to suppress surge, and a barrier around the edge of the disk to prevent overwash.

Meylan et al. (2015) presented measurements of the surge, heave and pitch motions of a thin plastic disk, as functions of $\lambda / D$. They used a disk with thickness over diameter quotient $H / D \approx 3.8 \times 10^{-2}$, and incident waves with lengths ranging from $\lambda / D \approx 0.9$ to 12.3 and steepness ranging from $k a \approx 0.01$ to 0.3. They compared the surge measurements to predictions of the slopesliding model. They showed the model predictions are accurate for incident wavelengths approximately greater than two floe diameters, for suitably chosen model parameters. However, they also used a barrier around the edge of the disk to prevent overwash. Thus, their findings do not imply the slope-sliding model will accurately predict the surge motion of a disk without a barrier. 
McGovern and Bai (2014) presented measurements of the heave and a com-

Further, the experimental measurements are compared to the surge motions predicted by the slope-sliding model, and the surge, heave and pitch motions predicted by the potential-flow/thin-plate model. The comparison is used to 


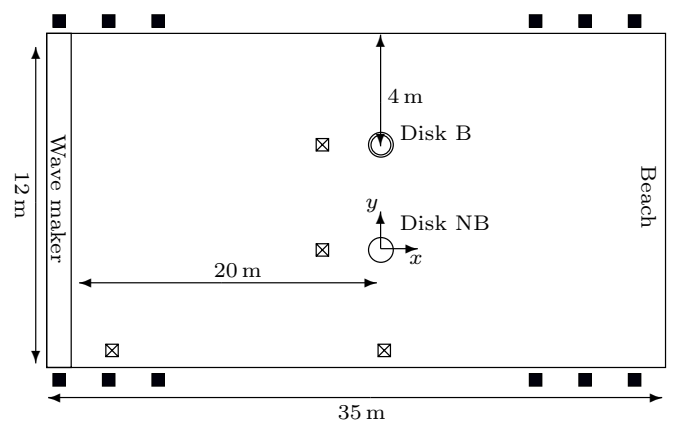

Figure 1: Schematic plan view of мтв. Wave probes $(\otimes)$ record the wave profiles at set locations, and motion tracking cameras ( $)$ record the motions of the disks.

estimate the limit of validity of the models and indicate phenomena they do not capture. The surge motions predicted by the two models are compared analytically and numerically in the long-wavelength regime.

\section{Experiments}

\subsection{Method}

Laboratory experiments were performed at the Australian Maritime College, Launceston, Australia, using the Model Test Basin (мтв) facility. During the experiments, the wave-induced motions of solitary floating disks were recorded. Figure 1 shows the plan view of the MTB and experimental set-up.

The Mтв is $35 \mathrm{~m}$ long and $12 \mathrm{~m}$ wide. It was filled with fresh water of density $\rho \approx 1000 \mathrm{~kg} \mathrm{~m}^{-3}$ to a depth of $h=0.83 \mathrm{~m}$. A piston-type wave maker bounds the MTB at its left-hand end. A sloping beach bounds the MTB at its right-hand end.

Locations in the MTB are defined by the Cartesian coordinate system $(x, y, z)$. The coordinate $(x, y)$ defines locations in the horizontal plane, parallel to the equilibrium water surface. The coordinate $x$ points from the wave maker to the beach. The coordinate $z$ defines the vertical location. It points upwards and its origin is set to coincide with the equilibrium water surface. 
Tests were conducted for a range of regular incident wave conditions. Target wave amplitudes from $a=2.5 \mathrm{~mm}$ to $50 \mathrm{~mm}$, and frequencies from $f=0.5 \mathrm{~Hz}$ to $2.0 \mathrm{~Hz}$, were tested. The corresponding wavelengths were approximately $\lambda=$ $0.4 \mathrm{~m}$ to $5 \mathrm{~m}$. The wavelengths are calculated as $\lambda=2 \pi / k$, where the wave number $k$ is the positive real root of the dispersion relation

$$
k \tanh k h=\kappa \quad \text { where } \quad \kappa=\frac{\omega^{2}}{g}
$$

is a frequency parameter, $\omega=2 \pi f$ is angular frequency, and $g \approx 9.81 \mathrm{~m} \mathrm{~s}^{-2}$ is gravitational acceleration.

Four wave probes were installed around the wave basin. The measured incident frequencies closely matched the target values. The measured incident amplitudes were generally slightly smaller than the target amplitudes. The results presented in $\S 2.3$ to $\S 4$ use the target frequencies and the measured amplitudes.

Tests were conducted for two matrices of incident wave amplitudes and frequencies. The first matrix contained more frequency entries than amplitude entries. The second matrix contained more amplitude entries. Table 1 summarises the tests conducted. Tests were not conducted for the largest amplitude and highest frequency combinations, to avoid wave breaking and non-planar waves. Non-planar waves were observed for incident wave steepnesses exceedeing $k a \approx 0.21$.

Two thin plastic Nycel disks were installed in the мтв. Nycel is an expanded rigid foam PVC. The disks had radii $R=200 \mathrm{~mm}$, thickness $H=15 \mathrm{~mm}$, density $\rho_{d} \approx 636 \mathrm{~kg} \mathrm{~m}^{-3}$, and hence equilibrium draft $d \approx 9.55 \mathrm{~mm}$ and mass $m=1.2 \mathrm{~kg}$.

An edge barrier was attached to one disk, which is referred to as Disk B. The disk with no barrier is referred to as Disk NB. The left-hand panel of Figure 2 shows a photo of Disk B. The edge barrier is a $50 \mathrm{~mm}$ high and $25 \mathrm{~mm}$ thick styrofoam ring. The barrier is used to prevent waves overwashing the surface of the disk, due to its small freeboard, and hence investigate whether the overwash affects disk motions.

The locations of the centres of mass of the disks at time $t$ are denoted 
$\underline{\text { Matrix } 1}$

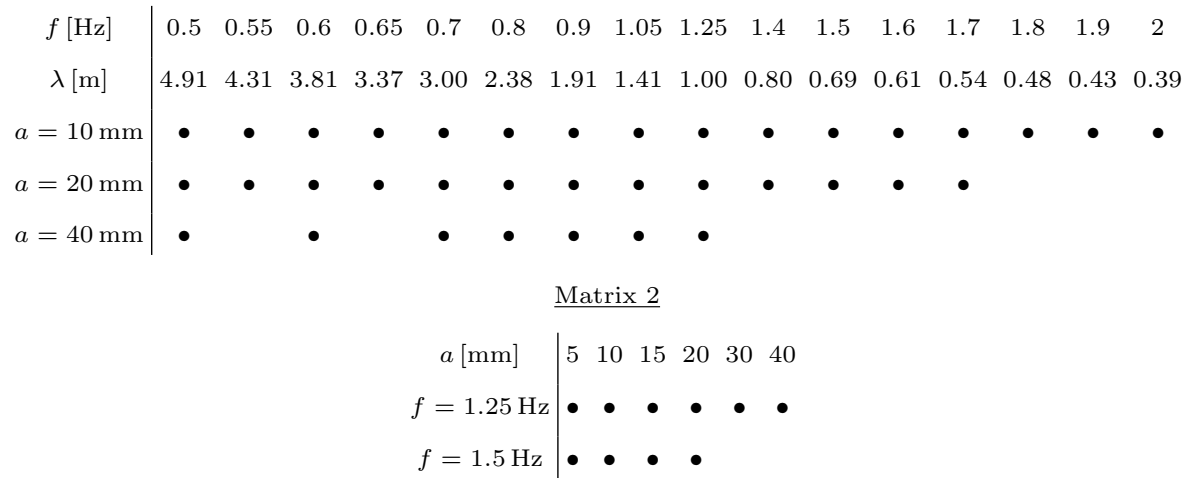

Table 1: Summary of test matrices.
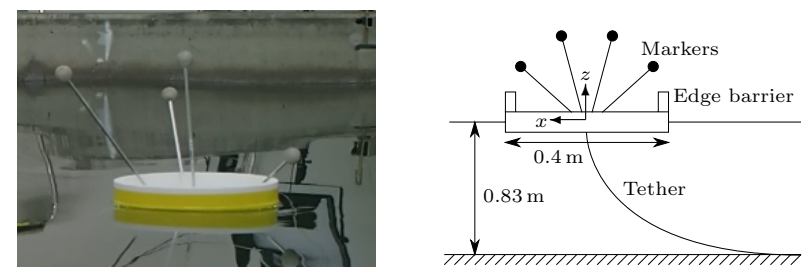

Figure 2: Left-hand panel: photo of Disk B. Edge barrier, and rods and markers are visible. Right-hand panel: cross-sectional schematic of Disk B, which shows the mooring system. 


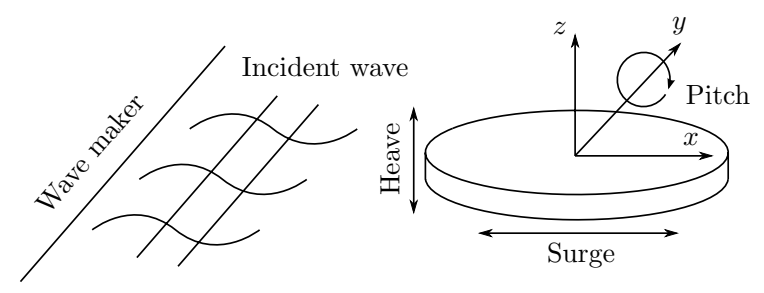

Figure 3: Schematic of Disk NB, including coordinate system and oscillatory motions.

$(x, y, z)=(X(t), Y(t), Z(t))$. The two disks were initially positioned approximately halfway down the basin. They were placed $4 \mathrm{~m}$ apart to minimise their scattered waves interfering with one another. Similarly, they were each placed

Motions in the six rigid-body degrees of freedom of the disks were recorded. The symmetric motions, with respect to the $x$-axis, were dominant due to the plane incident waves. No significant asymmetric motions were recorded, apart 

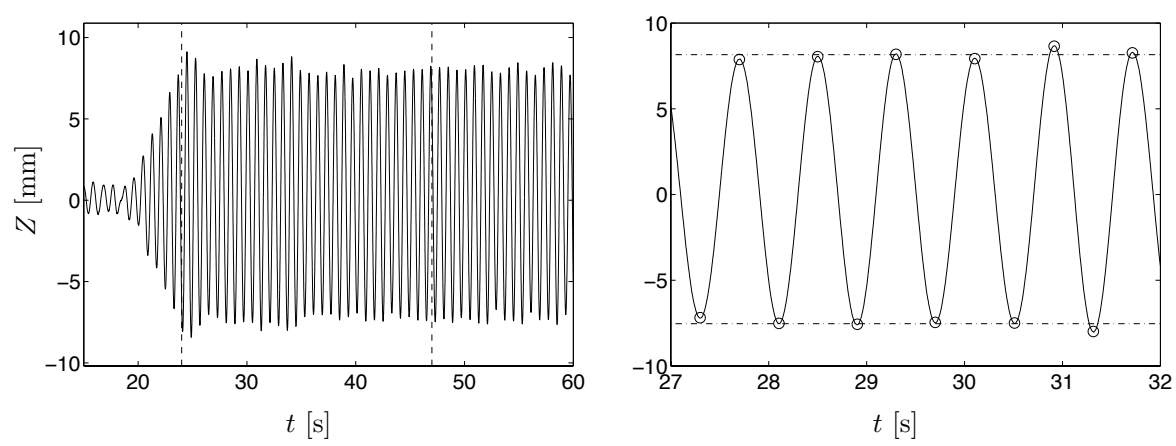

Figure 4: Left-hand panel: example motion in $z$-direction $(-)$. Steady-state interval is denoted by vertical lines (--). Right-hand panel: close-up of smoothed signal (-). Local maxima and minima (०) are shown. Mean maxima and minima are denoted by horizontal lines (---).

from, in some tests, long-period translational oscillations in the $y$-axis due to an initial offset with respect to the base of the tether. Other asymmetric motions (i.e. rotations about the $x$ - and $z$-axes) were, on average, less than $10 \%$ of the incident wave steepness.

The translational motion in the $x$-direction is a combination of drift, restoration due to the mooring system, and surge, which is an oscillatory motion at the frequency of the incident waves. The translational motion in the $z$-direction is oscillatory heave. The rotational motion in the $x z$-plane is oscillatory pitch. Figure 3 illustrates the oscillatory motions, which are analysed in this investigation.

\subsection{Data processing}

Figure 4 shows an example time series provided by Qualisys for the translational motion of Disk B in the $z$-direction. The test shown used an incident wave with frequency $f=1.25 \mathrm{~Hz}$ and measured amplitude $a=8.5 \mathrm{~mm}$.

Motions are considered within a steady-state interval, with respect to the oscillatory motions. This steady-state interval begins after the initial transient phase of the motions pass, and ends when waves reflected by the beach begin to interfere with the motions. The interval is approximated using the phase 
velocity of the wave, $c=f \lambda$. It is then refined manually.

The left-hand panel of Figure 4 shows the interval with vertical dotted lines. The interval runs from approximately $24 \mathrm{~s}$ to $45 \mathrm{~s}$, which is 26.25 incident wave periods. A consistent interval is used for analysis of all motions for a given test. The intervals used for the analysis have a mean length of approximately 23 wave periods, over all of the tests considered.

The MATLAB smooth function with the lowess method is used to eliminate noise from the raw signal and accurately identify the local maxima and minima. The smooth function applies a local regression method using a weighted linear least squares algorithm. The degree of smoothing is controlled by the smoothing parameter - a factor of 0.01 is specified here. The right-hand panel of Figure 4 shows the smoothed signal for a $5 \mathrm{~s}$ interval at the beginning of the steady-state 250 interval.

The maxima, minima, and their means are identified, as shown by the circles and horizontal dashed lines in the right-hand panel of Figure 4 The heave amplitude, $a_{H}$, is calculated as half the difference between the average peak and trough values. The same procedure is used to calculate the pitch amplitude, $a_{P}$, from the time series of the translational motion.

Note that in the example given in Figure 4 , the mean maxima and minima values are not centred about the zero ordinate axis. This is due to small disturbances, with amplitude of approximately $0.5 \mathrm{~mm}$, to the surface of the wave basin at the start of the test. However, this does not affect the calculation of the heave amplitude.

The left-hand panel of Figure 5 shows the translational motion in the $x$ direction, for the same test considered in Figure 4. Initially the disk drifts freely. The tether is engaged after it drifts approximately $60 \mathrm{~mm}$ down the basin. The combined drift and mooring forces create the long-period oscillation, which begins in the second half of the series shown. Surge is the relatively shortperiod oscillations superimposed on the free-drift and subsequent long-period oscillation.

The smooth function is used with a smoothing factor of 0.9 to extract the 

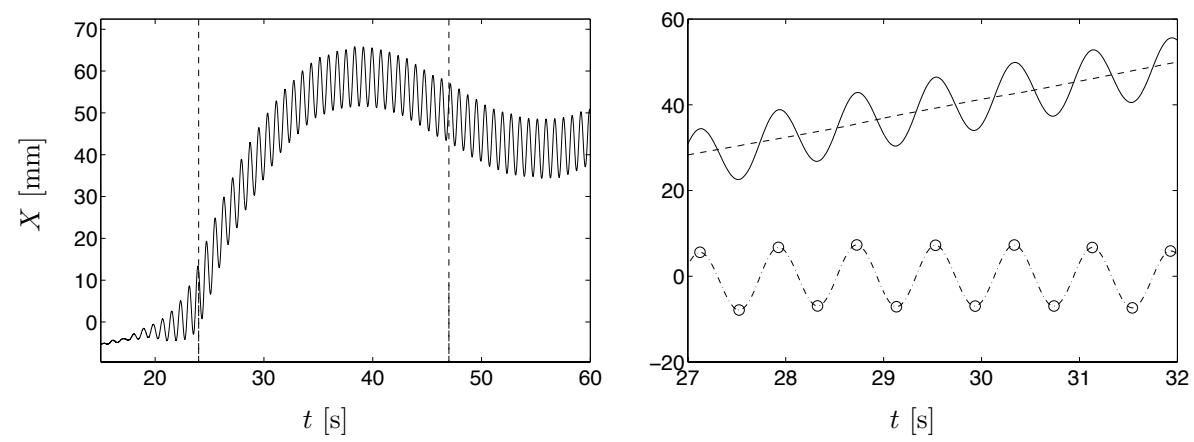

Figure 5: Left-hand panel: as in left-hand panel of Figure 4 but for motion in $x$-direction. Right-hand panel: close-up of smoothed motion (-), and decomposed into surge (--) and drift (--). Local maxima and minima of surge motion (o) are shown.

drift from the Qualysis data. The surge motion is the difference between the full data and the drift. The right-hand panel of Figure 5 illustrates the decomposition for the small time interval. The surge amplitude, $a_{S}$, is subsequently calculated using the same procedure as for heave and pitch.

For the example considered in Figure 5, the steady-state intervals span the interval of free drift and long-period oscillation. This occurs in most of the tests. Comparisons of surge amplitudes in the intervals of free drift and long-period oscillations show small differences only. For example, in Figure 5 , the average surge amplitudes sampled from $27 \mathrm{~s}$ to $32 \mathrm{~s}$ and $40 \mathrm{~s}$ to $45 \mathrm{~s}$ differ by less than $3 \%$. Similar results are observed in other tests. Further, note that the errors bars presented later accommodate effects of the mooring system on surge, pitch and heave.

\subsection{Response amplitude operators}

Figure 6 shows response amplitude operators (RAOs) of Disk NB for surge, heave and pitch, as functions of the incident wavelength nondimensionalised with respect to the disk diameter, $D=2 R$. The results are for the first test matrix. The surge RAO is $a_{S} /\{a \operatorname{coth} k h\}$, where $a \operatorname{coth} k h$ is the horizontal extent of the trajectory of a fluid particle at the free surface. The heave RAO 

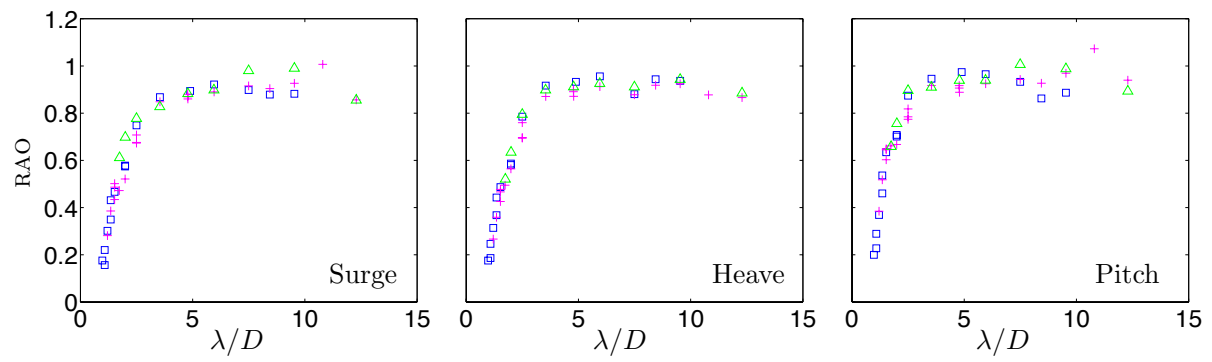

Figure 6: RAOs for Disk NB for Matrix 1, as functions of nondimensional incident wavelength. Data are grouped according to target incident wave amplitude: $a=10 \mathrm{~mm}(\square), 20 \mathrm{~mm}(+)$ and $40 \mathrm{~mm}(\triangle)$.
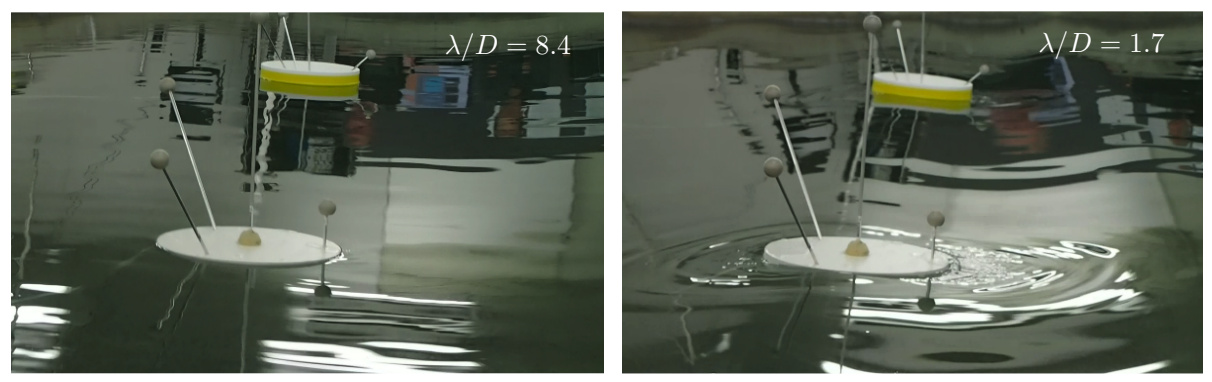

Figure 7: Example of changes to the wave field in the long- and short-wavelength regime (left-hand and right-hand panels, respectively).

is $a_{H} / a$. The pitch RAO is $a_{P} / k a$, where $k a$ is the incident wave steepness. In the sub-panels, data are grouped according to incident wave amplitude (using different symbols and colours). Error bars are omitted here for clarity. They are presented in Figures 13 to 15

Visually, the RAOs appear to be independent of the incident wave amplitude. The mean range of the corresponding RAOs for the different amplitudes is approximately 0.053 . Moreover, no behavioural trend with varying amplitude is evident. The results suggest a linear relationship exists between the amplitudes of oscillatory motion and the incident wave amplitude.

The RAOs are approximately unit valued for $\lambda / D$ greater than 3 . The disk does not affect the incident waves in this long-wavelength regime. The left-hand 

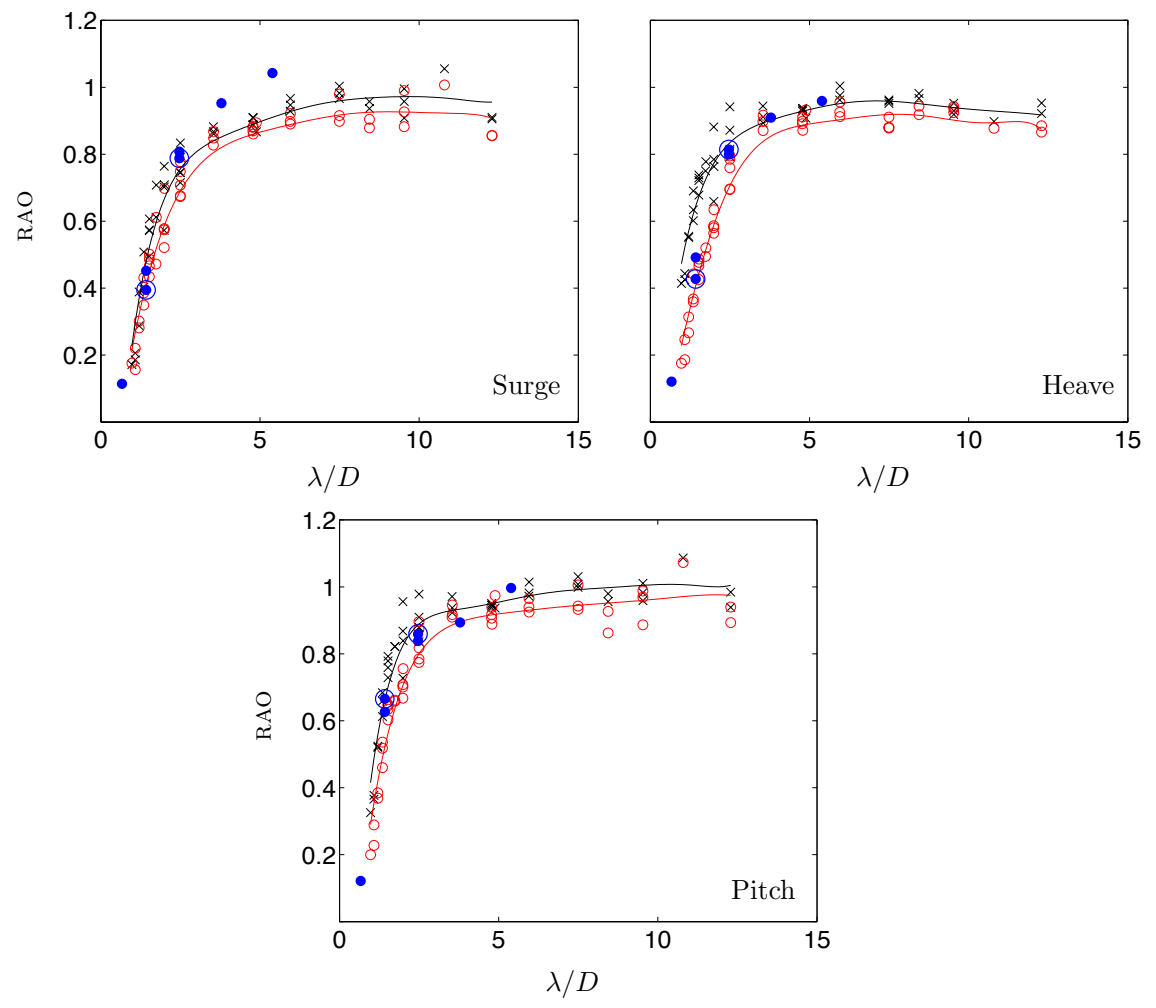

Figure 8: RAOs for Disk B $(\times)$ and Disk NB ( $\circ)$ for Matrix 1. Trend lines for Disk B $(-)$ and Disk NB (-) are shown. RAOs calculted by Bennetts and Williams (2015) are also included $(\bullet)$. Encircled bullets denote large-amplitude tests.

photo in Figure 7 shows an example of this behaviour. The disk simply follows the path of a fluid particle at the free surface.

The RAOs decrease as the incident wavelength decreases. The disks scatter the incident waves in the short-wavelength regime. The right-hand photo of Figure 7 shows an example of this. Scattering results in less energy being transferred into the oscillatory motions, and thus reduces the RAOs.

Figure 8 compares the RAOs of the two disks for the tests in the first matrix. Tests with different incident amplitudes are not distinguished from one another. Disk NB generally has slightly smaller RAOs than Disk B. The difference is greatest for heave at $16.4 \%$ and least for surge at $7.1 \%$. The percentages quoted 
represent the mean of the difference in the mean RAOs for the two disks, for an incident wavelength and RAO combination. Differences between the RAOs for the two disks are most pronounced in the short-wavelength regime, $\lambda / D<3$. The largest difference is 0.24 , which occurs for heave at $\lambda / D=0.9$.

Figure 8 also includes the RAO trend lines for each disk. Trend lines are generated using the mean RAOs at each incident wavelength. The smooth function is first applied to calculate a curve of best fit to the mean RAOs. The polyfit function is then used to generate a 7th-order polynomial approximations (trend lines), $p_{7}(\lambda / D)$, to the best fit curves. The trend lines are accurate to within $6 \%$ of the mean RAOs.

The relative change of the gradient of the trend lines, with respect to the incident wavelength, i.e. $p_{7}^{\prime \prime} / p_{7}^{\prime}$, where a prime denotes differentiation with re320 spect to $\lambda / D$, is used to quantify the transition from the strong dependence of the RAOs on the incident wavelength in the short-wavelength regime to insensitivity in the long-wavelength regime. For Disk NB the change in gradient has maxima of 1.14 at $\lambda / D \approx 2.2$ for surge, 1.21 at $\lambda / D \approx 2.5$ for heave and 1.74 at $\lambda / D \approx 2.7$ for pitch. For Disk $B$ the maxima are $0.86,1.14$ and 1.16 for surge, heave and pitch, respectively, all at at $\lambda / D \approx 3.2$. This indicates that the long-wavelength regime is attained for slightly longer incident wavelengths for the disk without a barrier, and that the transition is slightly sharper for the disk with the barrier.

Further, Figure 8 shows the RAOs calculated by Bennetts and Williams (2015). These are the only existing cognate results, as Montiel et al. (2013a b) used a vertical rod to prevent surge motion of the thin, flexible disks used in their experiments. Recall that Bennetts and Williams (2015) used a wooden disk without an edge barrier, with a thickness over diameter quotient $H / D=$ $3.3 \times 10^{-2}$ slightly less than the value $H / D=3.8 \times 10^{-2}$ for the dataset analysed 335 here. The density of the wooden disk was $\rho_{d} \approx 545 \mathrm{~kg} \mathrm{~m}^{-3}$, which is smaller than the density of the PVC disks $\rho_{d} \approx 636 \mathrm{~kg} \mathrm{~m}^{-3}$. Their disk was anchored to the floor via a series of springs and a steel cable.

The RAOs for the wooden disk are generally consistent with the PVC disks. 

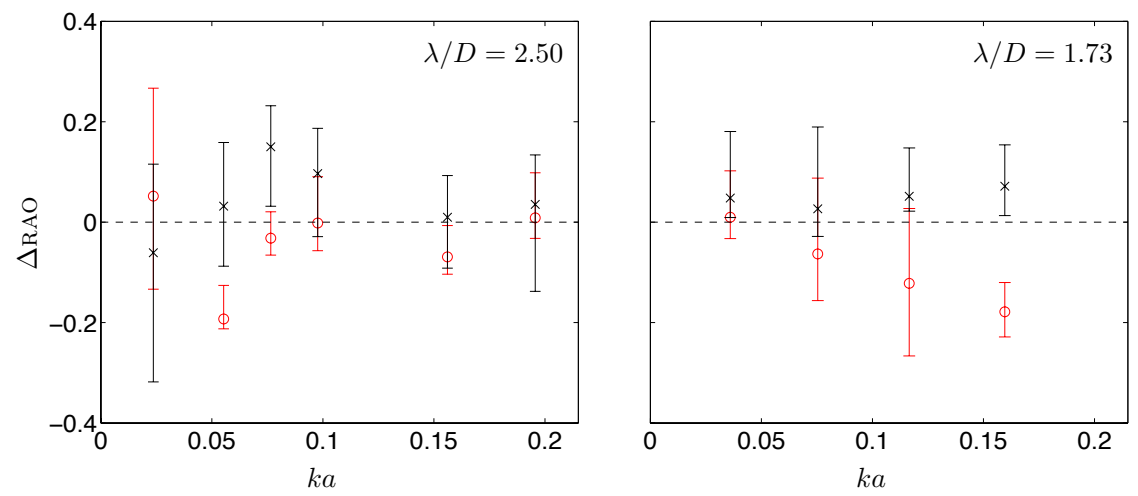

Figure 9: Deviation of surge RAO from the mean (--) for Matrix 2, as a function of wave steepness. Incident frequency is $f=1.25 \mathrm{~Hz}$ (left-hand panel) and $1.5 \mathrm{~Hz}$ (right). Results for Disk B $(\times)$ and Disk NB (०). Error-bar limits represent deviation of the RAOs corresponding to maximum and minimum individual amplitudes.

The only notable exceptions are the marginally larger surge RAOs for the two longest incident waves considered by Bennetts and Williams (2015), noting that this difference is within the error bars reported by Bennetts and Williams (2015) (not shown here) and those we present for our data in Figure 13. The RAOs for the wooden disk match the RAOs for Disk NB better than Disk B, especially for heave and pitch.

Figure 9 shows the surge RAO, as a function of the incident wave steepness, $k a$, calculated from tests in the second matrix. Surge motion is presented here, as it is the focus of this study. Cognate behaviours occur for heave and pitch motions (not shown).

The results are grouped according to the incident wavelength used (different panels) and which disk the results refer to (different symbols and colours). The RAOs are presented in terms of their relative deviation from the mean, defined by

$$
\Delta \mathrm{RAO}=\frac{\text { Surge RAO }-\langle\text { Surge RAO }\rangle}{\langle\text { Surge RAO }\rangle},
$$

where angled brackets $\langle\cdot\rangle$ denote the mean with respect to both disks and different steepnesses. The error bars denote RAO values for the maximum and 
minimum surge amplitudes in the tests.

For the longest incident wavelength tested, $\lambda / D=2.50$, the RAO values generally deviate by no more than $10 \%$ from the mean. The deviations here do not display a consistent trend and are, therefore, attributed to measurement errors. For the shortest wavelength tested, $\lambda / D=1.73$, the RAOs for Disk B are insensitive to steepness. In comparison, the RAOs for Disk NB decrease as steepness increases. This is attributed to the overwash phenomenon. Overwash becomes stronger as the incident amplitude, and hence steepness, increases.

The above finding is consistent with the amplitude dependence noted by Bennetts and Williams (2015) for $\lambda / D \approx 1.42$, shown in the left-hand and middle panels of Figure 8. In contrast to our finding, Bennetts and Williams (2015) results show pitch RAO marginally increases when the amplitude is doubled for $\lambda / D \approx 1.42$. However, Bennetts and Williams (2015) used two incident amplitudes only, and steepness for the largest amplitude was relatively low, $k a \approx 0.11$.

\section{Theoretical Models}

\subsection{Slope-sliding model}

The slope-sliding model assumes the floating body, here the disk, moves along the wave profile due to gravity. Its movement is resisted by drag between the body and water. The equation of motion in the $x$-direction is thus

$$
m\left(1+c_{m}\right) \frac{d V}{d t}=-m g\left[\frac{\partial \eta}{\partial x}\right]_{x=X}+\rho c_{d} l|\widehat{V}| \widehat{V}
$$

This is the version of the model derived by, for example, Grotmaack and Meylan (2006). It is a nonlinear ordinary differential equation, which is solved for the horizontal velocity of the disk, $V(t)=d X(t) / d t$.

The term on the left-hand side of Equation (3) is the inertial force of the disk. The coefficient $c_{m}$ is the added mass of the disk, i.e. its increased resistance to motion due to contact with water. The first term on the right-hand side is the sliding force due to gravity. The quantity $\eta(x, t)$ is the wave profile. The second term is the drag force. The quantity $\widehat{V}(t)=V_{w}(t)-V(t)$, where $V_{w}(t)$ is 

of the disk $x=X(t)$. The coefficient $c_{d}$ is the drag coefficient and $l=\pi R^{2}$ is the wetted surface area of the disk, noting that the slope-sliding model assumes the body sits on the wave surface, i.e. no submergence.

The model further assumes the disk diameter is small in relation to the incident wavelength. As discussed in $\S 2.3$ in this regime the incident wave profile is not modified by the disk, and is specified to be

$$
\eta(x, t)=a \sin (k x-\omega t)
$$

The phase of the incident wave does not affect the RAOs extracted from the model, and it is therefore normalised to the origin.

Following Grotmaack and Meylan (2006), using the wave profile (4) and the notation $Q(t)=k X-\omega t$, Equation 3 reduces to the autonomous dynamical system

$$
\frac{d V}{d t}=\frac{-m g k a \cos Q+\rho c_{d} l|\widehat{\mathcal{V}}| \widehat{\mathcal{V}}}{m\left(1+c_{m}\right)}
$$

where $\widehat{V}(t) \equiv \widehat{\mathcal{V}}(V, Q)=\omega a \sin Q \operatorname{coth} k h-V$, and

$$
\frac{d Q}{d t}=k V-\omega
$$

The system is defined on the surface of a cylinder, with azimuthal coordinate $0<Q<2 \pi$ and longitudinal coordinate $V \in \mathbb{R}$. Figure 10 shows the phase plane for an example problem, with zero drag and added mass, $c_{d}=c_{m}=0$, and an incident wave of amplitude $a=50 \mathrm{~mm}$ and length $\lambda=6 \mathrm{~m}$.

The zero-drag problem is analogous to the classic nonlinear pendulum model (e.g. Hirsch et al. 2013, § 9). It possesses two fixed points. For the example considered in Figure 10, with zero added mass, the fixed points are located at $(Q, V)=(\pi / 2, \omega / k)$ and $(3 \pi / 2, \omega / k)$. They can only be reached for an initial velocity, $V(0)$, comparable to that of the incident wave. The right-hand fixed point is a centre, i.e. the trajectories surrounding it are closed. The left-hand fixed point is a saddle node, which has lower and upper homoclinic connections. This is the so-called surfing solution. 


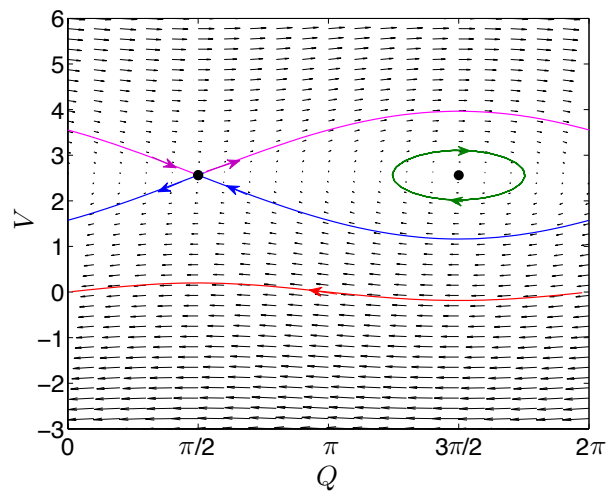

Figure 10: Phase diagram for slope-sliding model, using $\omega=2.68, c_{m}=c_{d}=0, a=0.05 \mathrm{~m}$ and $\lambda=6 \mathrm{~m}$. Vectors show the flow field. Two fixed points $(\bullet)$ exist at $(1.57,2.56)$ and $(4.71,2.56)$. The two curves emanating from the left-hand fixed point represent upper $(-)$ and lower (-) homoclinic connections. The elliptical curve centred around the right-hand fixed point $(-)$ represents a closed trajectory. The lower curve $(-)$ represents a periodic orbit.

Trajectories below the lower homoclinic connection in the phase plane are closed orbits. The periodic orbits represent disk motions consisting of a steady surge (causing the amplitude of the orbit) and drift (the difference in period of the orbit and the incident wave period). These are the relevant solutions for the present investigation. Figure 10 shows the orbit passing through the origin, i.e. a disk with zero initial velocity. The predicted surge motion is insensitive to perturbations of the initial condition.

For small non-zero values of the drag coefficient, the fixed points are shifted and the right-hand fixed point becomes a stable spiral. Trajectories below the lower homoclinic connection tend to the limit cycle, which oscillates around the $Q$-axis, i.e. it is analogous to the periodic orbit shown in Figure 10 . For large values of the drag coefficient, the fixed points disappear in a saddle-node bifurcation. All orbits then tend to the limit cycle.

In practice, the periodic orbit/limit cycle is obtained by solving system (5) as the second-order ordinary differential equation

$$
m\left(1+c_{m}\right) \frac{d^{2} X}{d t^{2}}+m g k a \cos (k X-\omega t)-\rho c_{d} l|\widehat{V}| \widehat{V}=0,
$$


with zero initial displacement and velocity, i.e. $X=d X / d t=0$ for $t=0$. The solution to 6 is obtained numerically using the MATLAB function ode45, which is based on a fourth and fifth-order Runge-Kutta method. For non-zero drag, the continuous function $\widehat{V} \tanh (100 \widehat{V})$ is used in place of the $|\widehat{V}|$. The steady solution, i.e. the periodic orbit/limit cycle, is decomposed into surge and drift components, as for the experimental data.

The values of the added mass and drag coefficients are obtained via comparison with experimental data. Meylan et al. (2015) compared the surge RAO obtained from the slope-sliding model to the experimental surge data for Disk B. They found the parameters $c_{m}=0.1$ and $c_{d}=0$ provided a good fit to the data for $\lambda / D>2$, approximately. For shorter incident wavelengths, the slope-sliding model does not capture the surge for any choice of added mass and drag coefficients.

\subsection{Linear potential-flow/thin-plate model}

The linear potential-flow/thin-plate model assumes the disk oscillates about its equilibrium position. The oscillations are time harmonic at the frequency of the incident wave, and are symmetric with respect to the $x$-axis, i.e. in-line with the incident wave. The location of the disk's centre of mass in the $x$-direction is denoted

$$
X(t)=\operatorname{Re}\left\{A_{S} \mathrm{e}^{-\mathrm{i} \omega t}\right\},
$$

where $A_{S}$ is the complex-valued surge amplitude, such that its magnitude is the surge amplitude, $a_{S}$, and its argument is the phase of surge motion. The vertical location of its lower surface oscillates about its equilibrium draught, and is denoted $Z(x, t)=-d+\operatorname{Re}\left\{w(x) \mathrm{e}^{-\mathrm{i} \omega t}\right\}$. The displacement function $w$ is decomposed as

$$
w(x)=A_{H}+A_{P} x+\sum_{n=1}^{\infty} \xi_{n} w_{n}(x) .
$$

Here $A_{H}$ and $A_{P}$ are complex-valued amplitudes of heave and pitch motions, respectively, such that $a_{H}=\left|A_{H}\right|$ and $a_{P}=\left|A_{P}\right|$. (Here, pitch is defined with respect to rotations around the geometric centre of the disk's lower surface, 
as opposed its centre of mass. This has a negligible effect for the thin disk considered.) Meylan and Squire (1996) originally proposed a decomposition of the form (8) for a thin floating disk. However, they neglected draught and surge, i.e. they set $d=A_{S}=0$. Bennetts et al. (2009) extended the disk model to include draught, and Montiel (2012, § 7.3) extended it to include surge.

The functions $w_{n}$ are the (symmetric) flexural modes of vibration and $\xi_{n}$ are the corresponding amplitudes. Itao and Crandall (1979) and Meylan and (1996) provide expressions for the flexural modes. For the incident wave and disk parameters studied here, the amplitudes $\xi_{n} \leq 5 \times 10^{-5} \mathrm{~mm}$, i.e. the disk responds to the incident waves in its rigid modes only.

Disk motions are forced by differential pressures from the surrounding water due to hydrodynamics. Air pressure is assumed to be constant, $p_{0}$ say. Water pressure is modelled using the linearised version of Bernoulli's equation

$$
p=p_{0}-\rho \frac{\partial \Phi}{\partial t}-\rho g z .
$$

Here $\Phi$ is the velocity potential of the water, i.e. the water velocity field is the spatial gradient of $\Phi$. Following potential-flow theory, it has been assumed the water is inviscid, homogeneous, incompressible and in irrotational motion.

Water motions are also assumed to be time harmonic, and the velocity potential is expressed as

$$
\Phi(x, y, z, t)=\operatorname{Re}\left\{\frac{g}{\mathrm{i} \omega} \phi(x, y, z) \mathrm{e}^{-\mathrm{i} \omega t}\right\} .
$$

The reduced (time independent) velocity potential, $\phi$, satisfies Laplace's equation throughout the water domain, i.e.

$$
\nabla^{2} \phi+\frac{\partial^{2} \phi}{\partial z^{2}}=0 \quad \text { where } \quad \nabla=(\partial / \partial x, \partial / \partial y)
$$

and a no-normal-flow condition on the floor of the basin, i.e.

$$
\frac{\partial \phi}{\partial z}=0 \quad \text { on } \quad z=-h .
$$

The water is assumed to extend to infinity in all horizontal directions, i.e. reflections from the basin boundaries are not considered. In the far-field, the velocity 
potential is composed of the incident-wave potential

$$
\phi_{I}(x, y, z)=\frac{a \mathrm{e}^{\mathrm{i} k x} \cosh k(z+h)}{\cosh k h},
$$

and a geometrically decaying scattered-wave potential, which satisfies the Sommerfeld Radiation condition

$$
\sqrt{r}\left(\partial_{r}-\mathrm{i} k\right)\left(\phi-\phi_{I}\right) \rightarrow 0 \quad \text { as } \quad r \rightarrow \infty
$$

where $r=\sqrt{\left\{x^{2}+y^{2}\right\}}$ is the radial coordinate.

The amplitudes of the waves and disk motions are assumed to be sufficiently small that linear theory is applicable. Conditions on moving boundaries are therefore approximated by linearised conditions applied on the corresponding equilibrium boundaries. The linearised free-surface condition is

$$
\frac{\partial \phi}{\partial z}=\kappa \phi \quad \text { on } \quad z=0
$$

where $\kappa$ is the frequency parameter defined in the second component of Equation (1). Equation 15 holds at all horizontal points $(x, y) \notin \Omega$, where $\Omega=\{x, y$ : $\left.x^{2}+y^{2}=r^{2} \leq R^{2}\right\}$ is the projection of the disk in equilibrium onto the $x y$-plane.

Following Kirchhoff-Love thin-plate theory (Timoshenko and WoinowskyKrieger, 1959), the linearised equation of motion in the $z$-direction is

$$
(1-\kappa d) w+F \nabla^{4} w=\phi \quad \text { on } \quad(x, y) \in \Omega, z=-d
$$

${ }_{440}$ Here $F=E H^{3} /\left\{12 \rho g\left(1-\nu^{2}\right)\right\}$ is the scaled flexural rigidity of the disk, where $E=530 \mathrm{MPa}$ is the Young's modulus, as measured by a three-point bending test on a strip of Nycel. The quantity $\nu=0.3$ is chosen as a typical value of Poisson's ratio for Nycel. Note, the model is insensitive to the elastic parameters, $E$ and $\nu$, as the disk responds rigidly.

The motion of the disk and the velocity potential are also coupled via the linearised kinematic conditions

$$
\frac{\partial \phi}{\partial r}=\kappa \cos (\theta) A_{S} \quad \text { on } \quad(x, y) \in \delta \Omega,-d<z<0,
$$


where $\theta=\tan (y / x)$ is the azimuthal coordinate and $\delta \Omega=\left\{x, y: x^{2}+y^{2}=R^{2}\right\}$ is the boundary of $\Omega$, and

$$
\frac{\partial \phi}{\partial z}=\kappa w \quad \text { on } \quad(x, y) \in \Omega, z=-d .
$$

Lastly, the vertical displacements of the disk satisfy the linearised free-edge conditions

$$
\begin{gathered}
\nabla^{2} w-(1-\nu)\left(\frac{1}{r} \frac{\partial w}{\partial r}+\frac{1}{r^{2}} \frac{\partial^{2} w}{\partial \theta^{2}}\right)=0 \quad \text { and } \\
\frac{\partial}{\partial r} \nabla^{2} w+(1-\nu) \frac{1}{r} \frac{\partial}{\partial r}\left(\frac{1}{r} \frac{\partial^{2} w}{\partial \theta^{2}}\right)=0,
\end{gathered}
$$

445 both for $(x, y) \in \delta \Omega$.

Equations (11) to (18) define a boundary value problem for the velocity potential, $\phi$. The vertical displacement of the disk, $w$, is obtained as part of the solution. At this juncture, the surge amplitude, $A_{S}$, is a parameter of the problem.

The velocity potential and vertical displacement function are decomposed as

$$
\phi=\hat{\phi}+A_{S} \check{\phi} \quad \text { and } \quad w=\hat{w}+A_{S} \check{w} \text {. }
$$

450 described by Linton and McIver (2001) for prototype hydrodynamic problems.

The surge amplitude, $A_{S}$, for the full problem is found via the linearised equation of motion of the disk in the $x$-direction, which is

$$
-\left(\omega^{2} m+\gamma_{m}\right) A_{S}=f
$$

The surge motion is forced by the pressure field around its edge created by the incident wave, i.e.

$$
f=\rho g R \int_{-d}^{0} \int_{-\pi}^{\pi} \cos (\theta)[\hat{\phi}]_{(x, y) \in \delta \Omega} \mathrm{d} \theta \mathrm{d} z .
$$




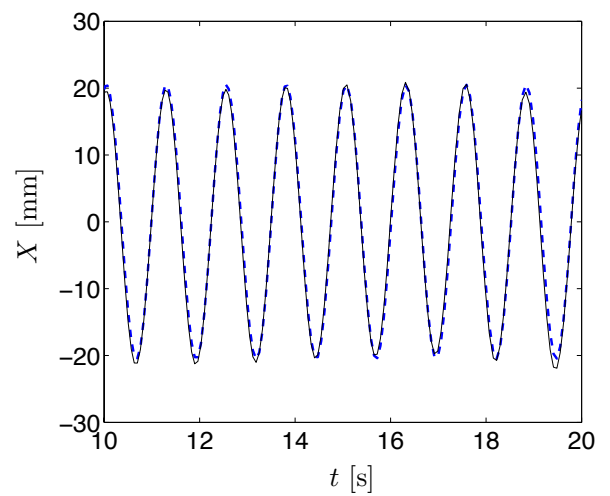

Figure 11: Slope-sliding model prediction of surge motion for $\lambda / D=6, a=20 \mathrm{~mm}$ and $c_{m}=c_{d}=0$. The surge component of the full numerical solution $(-)$ and the analytical long-wavelength solution (--) are shown.

The complex quantity $\gamma_{m}$ is defined by

$$
\gamma_{m}=\rho g R \int_{-d}^{0} \int_{-\pi}^{\pi} \cos (\theta)[\check{\phi}]_{(x, y) \in \delta \Omega} \mathrm{d} \theta \mathrm{d} z .
$$

The real and imaginary components of $\gamma_{m}$ are known as the damping and added mass terms, respectively (Mei 1983). The full solutions, $\phi$ and $w$, are obtained by substituting $A_{S}$ into Equation 19 .

\subsection{Long-wavelength regime}

At $\lambda / D=3$, which is approximately the transition between the short- and long-wavelength regimes, the slope-sliding model predicts that the drift velocity is $\mathrm{O}\left(10^{-3}\right) \mathrm{ms}^{-1}$. It decreases to $\mathrm{O}\left(10^{-4}\right) \mathrm{ms}^{-1}$ at $\lambda / D=12$. This holds for zero and non-zero values of the drag and added mass coefficients. Therefore, the slope-sliding model predicts the local (in time) horizontal motion of the disk is dominated by oscillatory surge in the large-wavelength regime.

Meylan et al. (2015) show that the slope-sliding model predictions of the RAOs are insensitive to drag. Neglecting drag, the slope-sliding model (3) is

$$
\begin{aligned}
\left(1+c_{m}\right) \frac{d V}{d t} & =-g\left[\frac{\partial \eta}{\partial x}\right]_{x=X} \\
& =-g a k \cos (k X-\omega t) .
\end{aligned}
$$


Assuming, without loss of generality, the disk is centred at the origin, the slow drift velocity is translated to the condition $|k X| \ll 1$. The slope-sliding model is, therefore, manipulated into the form

$$
\left(1+c_{m}\right) \frac{d V}{d t}=-g a k \cos \omega t+\mathrm{O}(k X),
$$

which, assuming zero initial velocity, is integrated to give

$$
V(t)=\frac{-g a k \sin (\omega t)}{\omega\left(1+c_{m}\right)}+\mathrm{O}(k X) .
$$

Thus, to leading order in $k X$, the displacement of the disk is

$$
X(t)=\frac{g a k \cos (\omega t)}{\omega^{2}\left(1+c_{m}\right)}=\frac{a \operatorname{coth}(k h) \cos (\omega t)}{1+c_{m}},
$$

where the final expression is derived using the dispersion relation. It follows that the slope-sliding model predicts the surge amplitude to be

$$
a_{S}=\frac{a \operatorname{coth}(k h)}{1+c_{m}}
$$

in the long-wavelength regime. Figure 11 shows the leading-order displacement (26) accurately approximates the surge motion of the full solution for $\lambda / D=6$, $a=20 \mathrm{~mm}$ and $c_{m}=c_{d}=0$.

The long-wavelength regime approximation that the disk does not modify the incident wave translates to $\hat{\phi} \approx \phi_{I}$ in the potential-flow/thin-plate model. Using this approximation, the forcing term, $f$, in the horizontal equation of motion 20 is

$$
\begin{aligned}
f & \approx \rho g R \int_{-d}^{0} \int_{-\pi}^{\pi} \frac{\cos (\theta) \mathrm{e}^{\mathrm{i} k R \cos \theta} \cosh k(z+h)}{\cosh k h} \mathrm{~d} \theta \mathrm{d} z \\
& =\mathrm{i} a g k \pi R^{2} d \rho+\mathrm{O}\left((k R)^{2}\right) .
\end{aligned}
$$

Therefore, to leading order in $k R$, the (complex-valued) surge amplitude, $A_{S}$, is

$$
A_{S}=\frac{-\mathrm{i} a g k m}{\omega^{2} m+\gamma_{m}},
$$

in which the Archimedean principle $m=\pi R^{2} d \rho$ has been used. Applying the dispersion relation, as before, it follows that the surge amplitude, $a_{S}=\left|A_{S}\right|$, is

$$
a_{S}=\frac{a \operatorname{coth} k h}{\left|1+q_{m}\right|}, \quad \text { where } \quad q_{m}=\frac{\gamma_{m}}{\omega^{2} m} .
$$



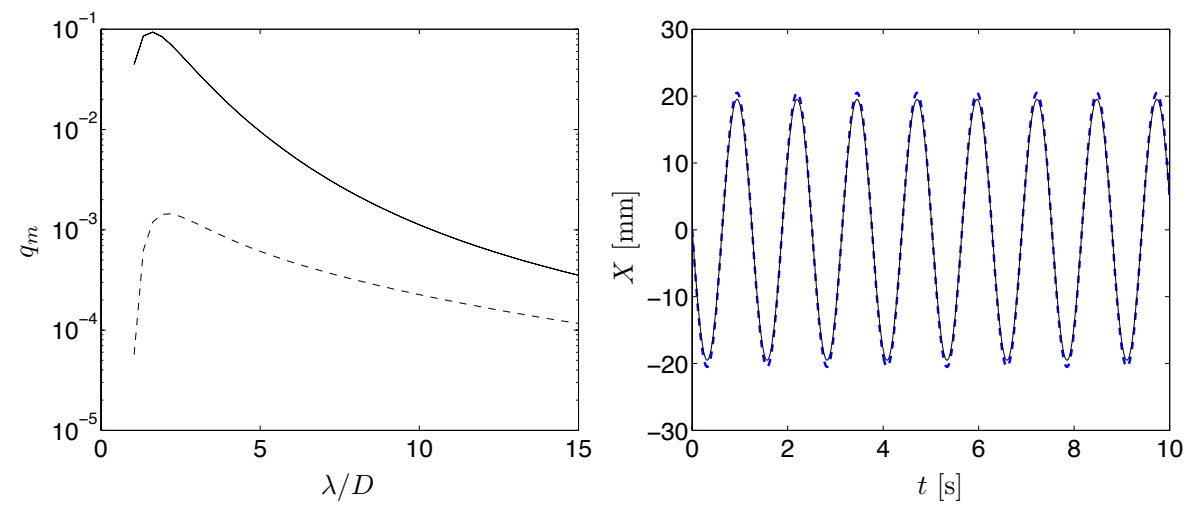

Figure 12: Left-hand panel: potential-flow/thin-plate model predictions of added mass (-) and damping (--) coefficients (i.e. imaginary and real parts of $q_{m}$, respectively), as functions of nondimensional incident wavelength. Right-hand panel: potential-flow/thin-plate model prediction of surge motions for $\lambda / D=6$ and $a=40 \mathrm{~mm}$. Full numerical solution (-) and analytical long-wavelength solution (--) are shown.

The potential-flow/thin-plate model therefore predicts a surge amplitude of the same form as the slope-sliding model in the long-wavelength regime. The models differ only in the second term on the denominator.

The left-hand panel of Figure 12 shows the real and imaginary parts of $q_{m}$, as functions of nondimensional wavelength. These two terms, which are scaled versions of the damping and added mass coefficients, respectively, are calculated from Equation (22). As expected, the figure shows that both coefficients tend to zero as $\lambda / D \rightarrow \infty$. The right-hand panel of Figure 12 shows the approximation with $q_{m}=0$ accurately captures the full solution of the potential-flow/thinplate model. The incident wave used in this example is the same as that used for Figure 11. Further, the analytical long-wavelength solution is within $10 \%$ of the full solution when $\lambda / D$ is approximately greater than 4 .

\section{RAOs: potential-flow/thin-plate model and data comparison}

The left-hand panels of Figures 13 to 15 show the RAOs predicted by the potential-flow/thin-plate model for surge, heave and pitch, respectively, as func- 

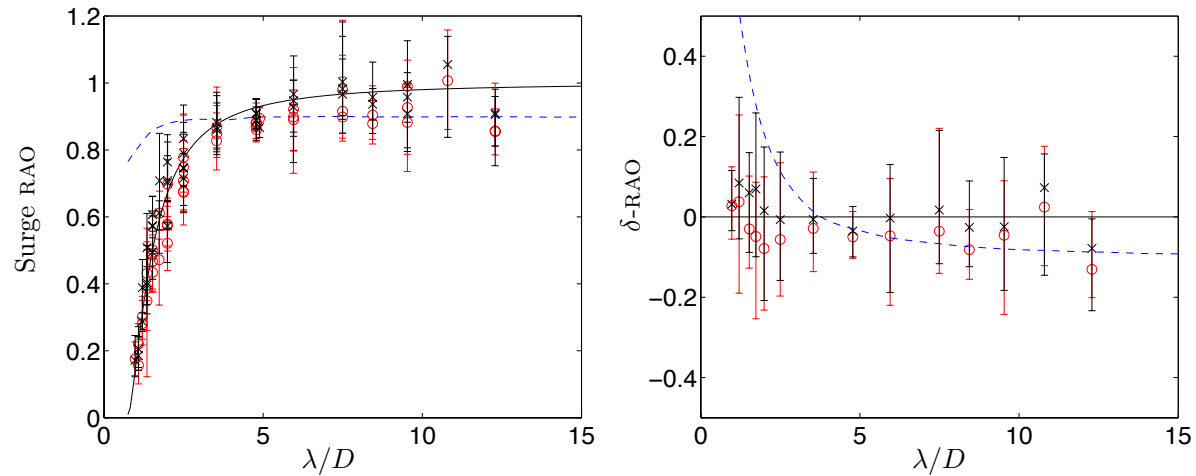

Figure 13: Left-hand panel: comparison of surge RAOs predicted by potential-flow/thin-plate model (-), the slope-sliding model with when $c_{d}=0$ and $c_{m}=0.1(--)$, and experimental data from Matrix 1 (Disk B, $\times$ and Disk NB, o). Error-bar limits represent RAOs for maximum and minimum amplitudes. Right-hand panel: differences between potential-flow/thin-plate model and slope-sliding model (--), and experimental data when grouped according to incident wavelength and disk (symbols and bars). Symbols represent group means. Error-bar limits represent overall group maximum and minimum differences.

tions of non-dimensional incident wavelength. Figure 13 includes the surge RAOs predicted by the slope-sliding model, using zero drag, $c_{d}=0$, and added mass $c_{m}=0.1$, as proposed by Meylan et al. (2015). The model predictions are overlaid on the RAOs calculated from the experimental data, as given in Figure 8. Error bars are included for the experimental results here. As in Figure 9, the upper and lower limits are calculated using maximum and minimum amplitudes for each test.

Visually, the potential-flow/thin-plate model predicts the mean RAOs measured during the experiments with pleasing accuracy over the range of incident wavelengths tested. Moreover, the surge RAO predictions lie within the error bars for all tests. The heave predictions only lie outside the error bars for Disk B when $\lambda / D<1.74$. The model consistently underpredicts the heave of Disk B in this regime. The pitch predictions generally lie inside the error bars, except for Disk NB when $\lambda / D<1.74$. The model consistently overpredicts the pitch of Disk NB in this regime. 

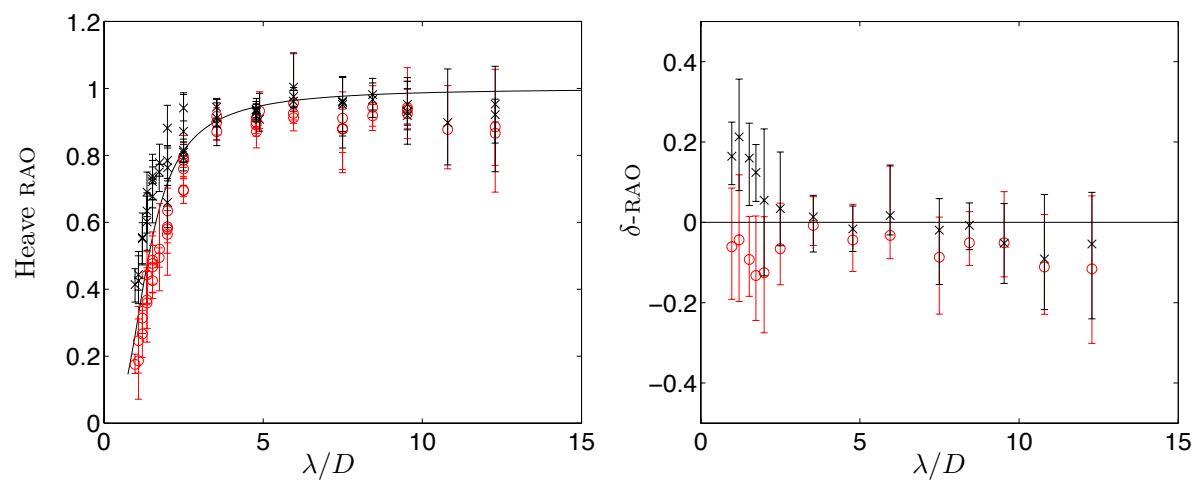

Figure 14: As per Figure 13 but for heave.
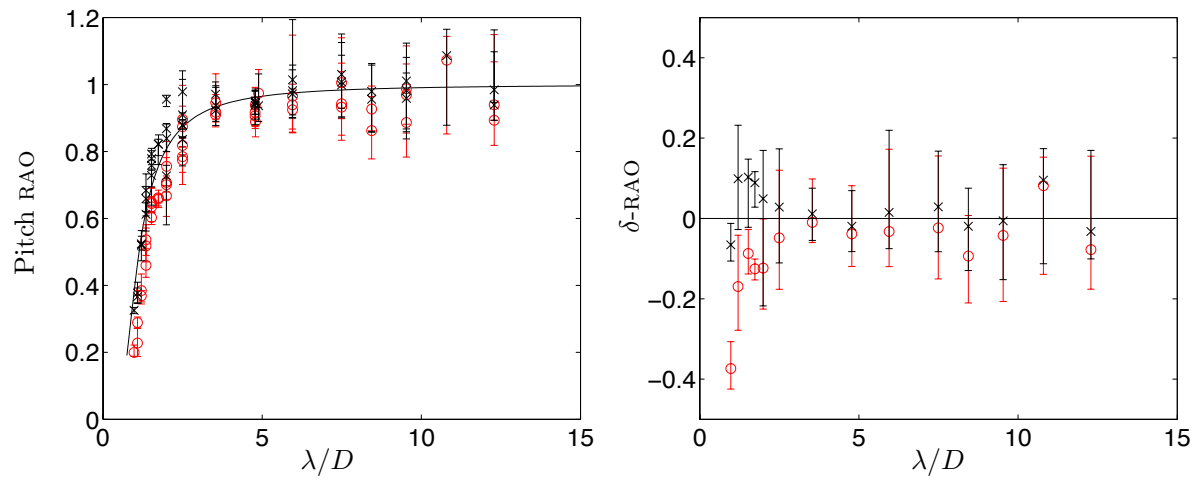

Figure 15: As per Figure 13 but for pitch. 
The slope-sliding model predicts the surge RAOs accurately in the long-

wavelength regime only, $\lambda / D \geq 3$. Note, however, that the high accuracy shown was achieved by tuning the added mass.

The right-hand panels of Figures 13 to 15 show the differences, $\delta$-RAO, between the RAOs calculated from the experimental data and predicted by the potential-flow/thin-plate model. Here, the experimental data are grouped according to the incident wavelength and the disk type. The symbols denote the group means for each disk. The error bars represent the overall maximum and minimum differences for each group, i.e. the maximum and minimum for the RAOs calculated from the maximum and minimum individual amplitudes.

For Disk B, the largest mean difference, with respect to incident wavelength, between the experimental group means and the potential-flow/thin-plate model is 0.04 units for heave, and the least is 0.01 units for surge. For Disk NB, the largest difference is -0.07 units for heave, and the least is -0.04 units for surge.

Differences between the model and data are generally larger for short incident wavelengths than long incident wavelengths. For instance, for the heave RAO for Disk B, the mean difference is 0.13 units for $\lambda / D<3$ and 0.02 units for $\lambda / D>3$. There is a clear tendency for the model to overpredict the RAOs of Disk B in the short-wavelength regime, and underpredict its RAOs in the shortwavelength regimes. In comparison, the model tends to overpredict the RAOs of Disk NB in both regimes.

Figure 13 includes the difference between the surge RAOs predicted by the slope-sliding and potential-flow/thin-plate models. The slope-sliding model predicts smaller RAOs than the potential-flow/thin-plate model in the longwavelength regime. The difference reaches an asymptotic value, as indicated by the analysis of $\S 3.3$. The slope-sliding model predicts larger RAOs than the potential-flow/thin-plate model in the short-wavelength regime. The difference increases monotonically as the incident wavelength decreases, as the surge RAOs predicted by the potential-flow/thin-plate model are decreasing more rapidly than those of the slope-sliding model in this interval. This behaviour will not persist for smaller incident wavelengths as the potential-flow/thin-plate model 

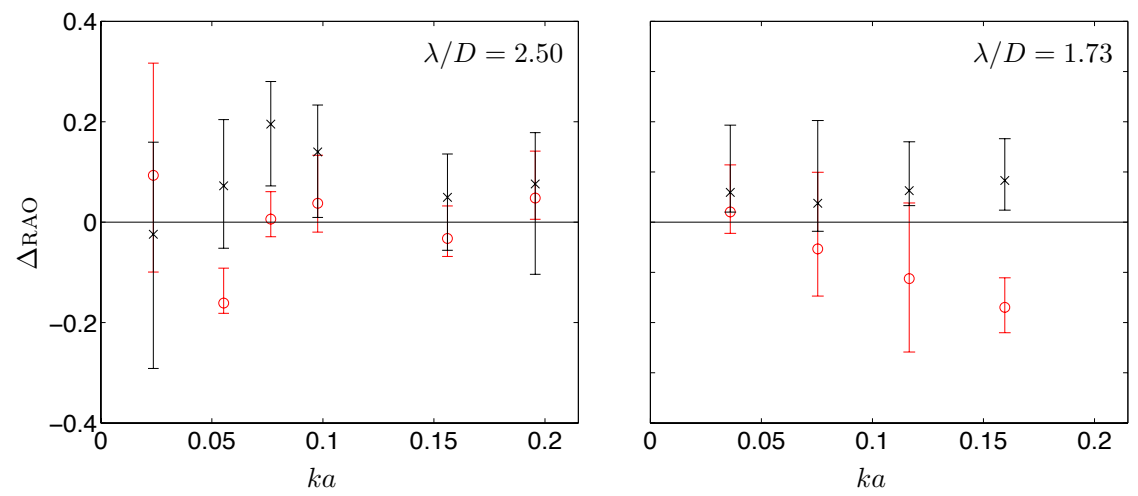

Figure 16: As per Figure 9, but for deviation of experimental data from disk model.

predicts a surge RAO of approximately zero for the smallest wavelength considered here.

Figure 16 shows the deviation of the experimental data for surge from the potential-flow/thin-plate model, using data from the second test matrix. The deviation is defined and presented as in Figure 9 , but with the model predictions replacing the mean. The model generally underpredicts the measured surge RAOs for the longer wavelength, $\lambda / D=2.50$, noting that this value is close to the transition between the short- and long-wavelength regimes. There are two exceptions, both for Disk NB. The mean deviation is $11.5 \%$ for Disk B and $2.1 \%$ for Disk NB. The model, therefore, predicts the surge of the disk without a barrier more accurately than the disk with a barrier. The model also underpredicts the surge motion of Disk B for the shorter wavelength, $\lambda / D=$ 1.78. The deviation for Disk B has mean 9.7\%. The model underpredicts the surge of Disk B for the lowest steepness only. The decrease in surge RAOs for Disk NB with increasing steepness, noted in $\S 2.3$, results in the model overpredicting the surge motion, with the deviation from the RAO up to $-15.8 \%$ for the steepest wave considered. 


\section{Summary and discussion}

Results from a series of laboratory wave basin experiments were reported.

In the experiments the motions of two solitary, floating, thin, plastic disks were measured. The motions were forced by regular incident waves. Tests were conducted for incident wavelengths ranging from approximately one to 12 times the disk diameter, and for a range of incident amplitudes. One disk had a barrier attached to its edge to prevent waves overwashing it. It was used to infer the impact of overwash on the motions of the disk without a barrier. Amplitudes of the dominant oscillatory motions were extracted from the recorded time series. They were presented as response amplitude operators (RAOs). Particular attention was given to the surge RAO.

The key findings were:

1. The disks move like a particle on the water surface for incident wavelengths approximately three times greater than the disk diameter. This ratio was used to define the transition between the short- and long-wavelength regimes.

2. For the disk with a barrier, the surge, heave and pitch RAOs are insensitive to the incident wave amplitude.

3. The disk without a barrier's RAOs are slightly smaller than those of the disk with a barrier. The differences are most pronounced in the shortwavelength regime and for heave motion.

4. For a relatively short incident wavelength, the disk without a barrier's RAOs decrease as the incident amplitude increases. For a larger incident wavelength, the RAOs are insensitive to the incident amplitude.

The amplitude dependence occurring only for the disk without a barrier implies that it is related to the overwash phenomenon. The presence of overwash on the disk is likely to suppress its motions. Moreover, the overwash is generated at the disk edge in lieu of a surface for the wave to apply pressure to. The absence of this surface is also likely to reduce the disk motions. 
The experimental study was motivated by theoretical models of wave-induced motions of sea ice floes, which often model floes as thin floating disks. Two theoretical models were considered here. The first model is based on slope-sliding theory, in which gravity pulls the disk down the wave profile. The second model is based on combined linear potential-flow and thin-plate theories, in which disk motions are forced by differential pressure around the wetted surface of the disk.

It was shown analytically that the two different theories predict the same form for the surge amplitude in the long-wavelength regime. The model predictions differ only in the added mass terms used. The potential-flow/thin-plate model predicts the added mass tends to zero in the long-wavelength regime. The added mass is user set in the slope-sliding model. It therefore retains this extra unknown as a tuning parameter.

The RAOs predicted by the models were compared to the RAOs extracted from the experimental data. The key findings were:

1. The potential-flow/thin-plate model captures the RAOs with pleasing accuracy, when viewed as a function of incident wavelength. The model is most accurate for surge and least accurate for heave.

2. In the long-wavelength regime, the potential-flow/thin-plate model tends to overpredict the RAOs. In the short-wavelength regime, it tends to underpredict the RAOs of the disk with a barrier and overpredict those of the disk without a barrier. The latter property is exacerbated by increasing the incident amplitude, which, as noted above, decreases the RAOs.

3. The slope-sliding model captures the surge RAO accurately in the longwavelength regime, with appropriate tuning of the added mass.

The findings imply that linear potential-flow/thin-plate theory is viable to model the oscillatory motions of a thin floating disk. However, a certain degree of caution is necessary. In the long-wavelength regime the model slightly overpredicts the RAOs, although within the error bounds for the measurements. The additional tuning parameter available in the slope-sliding theory provides improved model-data agreement for surge. In the short-wavelength regime and for 
moderate incident amplitudes, the findings imply that extending the potentialflow/thin-plate model to incorporate overwash will improve its accuracy, noting that Skene et al. (2015) recently showed that the potential-flow/thin-plate model can be used to predict overwash depths when the overwash is relatively shallow.

The Australian Research Council funds LJY's PhD Scholarship and provides funding support to LGB (DE130101571). The Australian Antarctic Science Program provides support for LGB (Project 4123). The U.S. Office of Naval Research supports MHM. The Australian Maritime College funded the experiments. The authors thank Tim Lilienthal and Kirk Meyer for technical support during the experiments.

\section{References}

\section{References}

Bennetts, L.G., Biggs, N.R.T., Porter, D., 2009. Wave scattering by an axisymmetric ice floe of varying thickness. IMA J. Appl. Math. 74, 273-295.

Bennetts, L.G., Peter, M.A., Squire, V.A., Meylan, M.H., 2010. A threedimensional model of wave attenuation in the marginal ice zone. J. Geophys. Res. 115, C12043.

Bennetts, L.G., Squire, V.A., 2012. On the calculation of an attenuation coefficient for transects of ice-covered ocean. Proc. R. Soc. Lond. A 468, 136-162.

Bennetts, L.G., Williams, T.D., 2015. Water wave transmission by an array of floating disks. Proc. R. Soc. Lond. A 470.

Dai, M., Shen, H.H., Hopkins, M.A., Ackley, S.F., 2004. Wave rafting and the equilibrium pancake ice cover thickness. J. Geophys. Res. 109, C07023.

Grotmaack, R., Meylan, M.H., 2006. Wave forcing on small floating bodies. J. Waterway, Port, Coastal, Ocean Eng. 132, 192-198. 
Harms, V.W., 1987. Steady wave-drift of modeled ice floes. J. Waterway, Port, Coastal, Ocean Eng. 113, 606-622.

Hirsch, M.W., Smale, S., Devaney, R.L., 2013. Differential equations, dynamical systems and an introduction to chaos. 3rd ed., Elsevier.

Huang, G., Law, A.W.K., Huang, Z., 2011. Wave-induced drift of small floating objects in regular waves. Ocean Engineering 38, $712-718$.

Isaacson, M., 1982. Fixed and floating axisymmetric structures in waves. J. Waterw. Port. Coastal and Ocean Div. , 180-199.

640 Itao, K., Crandall, S.H., 1979. Natural modes and natural frequencies of uniform, circular, free-edge plates. J. Appl. Mech. 46, 448-453.

Kohout, A.L., Meylan, M.H., 2008. An elastic plate model for wave attenuation and ice floe breaking in the marginal ice zone. J. Geophys. Res. 113.

Langhorne, P.J., Squire, V.A., Haskell, T.G., 2001. Lifetime estimation for a fast ice sheet subjected to ocean swell. Annals Glaciol. 33, 333-338.

Linton, C.M., McIver, P., 2001. Handbook of Mathematical Techniques for Wave/Structure Interactions. Chapman \& Hall/CRC.

Marchenko, A.V., 1999. The floating behaviour of a small body acted upon by a surface wave. J. Appl. Math. Mech. 63, 471-478.

Martin, S., Becker, P., 1987. High-frequency ice floe collisions in the Greenland Sea during the 1984 marginal ice zone experiment. J. Geophys. Res. 92, 7071-7084.

Massom, R., Stammerjohn, S., 2010. Antarctic sea ice variability: Physical and ecological implications. Polar Sci. 4, 149-458.

655 Masson, D., LeBlond, P., 1989. Spectral evolution of wind-generated surface gravity waves in a dispersed ice field. J. Fluid Mech. 202, 111-136. 
McGovern, D., Bai, W., 2014. Experimental study on kinematics of sea ice floes in regular waves. Cold Reg. Sci. Technol. 103, 15-30.

McKenna, R.F., Croker, G.B., 1990. Wave energy and floe collisions in marginal ice zones, in: Murphy, T.K.S. (Ed.), Ice Technology for Polar Regions, proceedings of the Second International Conference on Ice Technology, Computational Mechanics Publications, Southampton. pp. 33-41.

Mei, C.C., 1983. The Applied Dynamics of Ocean Surface Waves. John Wiley \& Sons, Inc.

Meylan, M.H., 2002. Wave response of an ice floe of arbitrary geometry. J. Geophys. Res. 107.

Meylan, M.H., Squire, V.A., 1996. Response of a circular ice floe to ocean waves. J. Geophys. Res. 101, 8869-8884.

Meylan, M.H., Squire, V.A., Fox, C., 1997. Towards realism in modeling ocean 670 wave behavior in marginal ice zones. J. Geophys. Res. 102, 22981-22991.

Meylan, M.H., Yiew, L.J., Bennetts, L.G., French, B.J., Thomas, G.T., 2015. Surge motion of an ice floe in waves: comparison of theoretical and experimental models. Annals Glaciol. 56, 107-111.

Montiel, F., 2012. Numerical and experimental analysis of water wave scattering 675 by floating elastic plates. Ph.D. thesis. University of Otago. Dunedin, New Zealand.

Montiel, F., Bonnefoy, F., Ferrant, P., Bennetts, L.G., Squire, V.A., Marsault, P., 2013a. Hydroelastic response of floating elastic disks to regular waves. Part 1: Wave tank experiments. J. Fluid Mech. 723, 604-628.

Montiel, F., Bennetts, L.G., Squire, V.A., Bonnefoy, F., Ferrant, P., 2013 b. Hydroelastic response of floating elastic disks to regular waves. Part 2: Modal analysis. J. Fluid Mech. 723, 629-652. 
Prinsenberg, S.J., Peterson, I.K., 2011. Observing regional-scale pack-ice decay processes with helicopter-borne sensors and moored upward-looking sonars. Annals Glaciol. 52, 35-42.

Rumer, R.R., Chrissmas, R.D., Wake, A., 1979. Ice Transport in Great Lakes. Number 79-3 in Water Resources and Environmental Engineering Research, NY State Univ. at Buffalo.

Shen, H.H., Ackley, S.F., 1991. A one-dimensional model for wave-induced ice-floe collisions. Annals Glaciol. 15, 87-95.

Shen, H.H., Zhong, Y., 2001. Theoretical study of drift of small rigid floating objects in wave fields. J. Waterway, Port, Coastal, Ocean Eng. 127, 343-351.

Skene, D.M., Bennetts, L.G., Meylan, M.H., Toffoli, A., 2015. Modelling water wave overwash of a thin floating plate. J. Fluid Mech. 777, R3.

Squire, V.A., 2007. Of ocean waves and sea-ice revisited. Cold Reg. Sci. Technol. $49,110-133$.

Squire, V.A., 2011. Past, present and impendent hydroelastic challenges in the polar and subpolar seas. Phil. Trans. R. Soc. Lond. A 369, 2813-2831.

Squire, V.A., Dugan, J.P., Wadhams, P., Rottier, P.J., Liu, A.K., 1995. Of ocean waves and sea ice. Annu. Rev. Fluid Mech. 27, 115-168.

Squire, V.A., Moore, S.C., 1980. Direct measurement of the attenuation of ocean waves by pack ice. Nature 283, 365-368.

Stroeve, J., Markus, T., Boisvert, L., Miller, J., Barrett, A., 2014. Changes in Arctic melt season and implications for sea ice loss. Geophys. Res. Lett. 41, $1216-1225$.

Strong, C., Rigor, I.G., 2013. Arctic marginal ice zone trending larger in summer and narrower in winter. Geophys. Res. Lett. 40, 4864-4868. 
Thomson, J., Rogers, W.E., 2014. Swell and sea in the emerging Arctic Ocean. Geophys. Res. Lett. 41, 3136-3140.

${ }_{710}$ Timoshenko, S., Woinowsky-Krieger, S., 1959. Theory of Plates and Shells. 2nd ed., McGraw-Hill, New York.

Wadhams, P., 1983. A mechanism for the formation of ice edge bands. J. Geophys. Res. 88, 2813-2818.

Wadhams, P., Gill, A.E., Linden, P.F., 1979. Transects by submarine of the East Greenland Polar Front. Deep Sea Res. 26, 1311-1327.

Williams, T.D., Bennetts, L.G., Dumont, D., Squire, V.A., Bertino, L., 2013a. Wave-ice interactions in the marginal ice zone. Part 1: Theoretical foundations. Ocean Model. 71, 81-91.

Williams, T.D., Bennetts, L.G., Dumont, D., Squire, V.A., Bertino, L., 2013 b.

Wave-ice interactions in the marginal ice zone. Part 2: Numerical implementation and sensitivity studies along $1 \mathrm{~d}$ transects of the ocean surface. Ocean Model. 71, 92-101. 